\title{
ANALYTICAL EXPRESSIONS OF RISKS INVOLVED IN GENERAL INSURANCE
}

The following comments on the papers written in English, French and German under Subject IVA and published in Trans. XVth. International Congress of Actuaries I957, formed the basis of an address by Mr. C. Philipson, Sweden, on Thursday I7th October, to the members of Astin in New York.

Franckx, Belgium - "Bornes du risque dans l'assurance élémentaire"

Let $s_{n}$ be the maximum value of $s_{i}$ and $t_{n}$ the minimum value of the angular coefficient of the straight line connecting the point $\left(s_{i}, A_{i}\right)$ with point $(\mathrm{I}, \mathrm{I})$, when $i$ takes the values $\mathrm{I}, 2 \ldots \mathrm{n}$ and the point $\left(s_{i}, A_{i}\right)$ is taken to mean the vertex of the convex curve representing the generating function of the $i$ th favourable risk. In a portfolio of $m+n$ independent risks-where $\mathrm{m}$ are unfavourable and $n$ favourable-Franckx finds that the superior limit of the probability that the aggregate amount of claims shall not exceed $k$ can be written

$I_{m} \frac{\left[\mathrm{I}-\left(\mathrm{I}-s_{n}\right) t_{n}\right]^{n}}{s_{n}^{k}}$, where $I_{m}$ is taken to mean the product of the generating functions for the $\mathrm{m}$ unfavourable risks.

Speaking of the theory of risk as first given by Filip Lundberg and developed by Cramér and the Scandinavian School he writes:

"Cela n'empêche qu'il y a encore un monde entre la théorie et la pratique et que les actuaires n'auront la partie gagnée que dans la mesure ou leurs travaux seront suffissamment simples que pour qu'ils soient et compréhensibles et directement utilisables".

An example of the direct application of the theory of Risk is the following paper by one of Cramér's pupils.

Segerdahl, Sweden - "If a risk business goes bankrupt, when does it occur?"

The writer gives numerical computations of the parameters of the well-known approximation formula for the probability of ruin (invol- 
ving the loading $\lambda$, the parameter $C$ being a function of $\lambda$, and the intensity $R$ ) for three fixed values of $R$. He also cadculates the first two moments of the distribution in time of the first point of ruin. The calculations are carried out for three different assumptions for the distribution of risk sums, called "equal", "exponential" and "fire". The "equal" distribution can be regarded as representing industrial life insurance and the "exponential" ordinary life insurance. The "fire" distribution is based on an experience from Swedish non-industry fire insurance over the years I948-5I.

As an example taken from Segerdahl's paper, a level of $R=$ $5 \%$ corresponds to values of $\lambda=2.5,5.0$ and $157.7 \%$ and values of $C=.998$, .995 and .676 for the "equal", "exponential" and "fire" distributions respectively.

The difference between life and general insurance, as shown by the difference in $\lambda$, is due to the properties of the distribution of risk sums. In the case of life insurance this represents mainly the distribution of the sums insured and of ages within the company, whilst as regards general insurance the variation in the degree of damage is generally also involved.

Johansen, Denmark - "On fire insurance of rural buildings" and Benktander, Sweden - "On the variation of the risk premium with the dimensions of the house within fire insurance".

These two authors deal with simple cases of fire insurance, where the deviation of the distribution of the risk sum from an equal distribution can be neglected.

Johansen, observing that farm buildings when ignited will either be heavily or only slightly damaged, assumes that as far as such buildings are concerned we may base our premium calculation on the concept of total fires only. The probability of ignition is supposed to be proportionate to the sum insured, i.e. the latter multiplied by a factor dependent on the nature of the risk. The consequence of the assumption is that the risk premium is proportionate to the square of the sum insured or the risk premium rate proportionate to the surn insured. The assumptions are tested with the Danish tariffs for farm buildings and it is found that the agreement between the theory and the test material is extremely good. The theory is used for calculation of premiums for two or more buildings 
where the probabilities of ignition are more or less dependent.

Benktander has proved in earlier papers that as far as the experience investigated is concerned the ignition frequency can be represented by a linear function of the sum insured; for agricultural outbuildings the risk premium rate was found to be linearly dependent on the sum insured. The risk premium rates determined from single years are analysed in two ways. Firstly, for a risk group characterised by the parameters $\alpha$ and $\beta$ the rate $\gamma_{s}^{(i)}$, for the year $i$ and the sum insured $s$ can be written

$$
r_{s}^{(i)}=k_{i}\left(\alpha+\frac{\bar{s}}{s_{i}} \beta . s\right) \text {, where } k_{i} \text { denotes the risk premium }
$$

level of the year $i$ and the ratio between the mean insurance sum for the whole observation period $(\bar{s})$ and for the year $i,\left(s_{i}\right)$, represents an "inflation" factor.

Secondly the risk premium rate is considered to be formed by. contributions from different causes of fire. Some of these causes, of which those connected with heating and those dependent on ignition from other risks are mentioned, give contributions which are independent of $s$. Others, comprising causes not investigated and lightning are dependent on the sum insured; the fluctuations in the incidence of lightning produce a variation in the rate proportionate to the total rate and therefore some proportion of $k_{i}$ may be regarded as the contribution from lightning.

Benckert and Sternberg, Sweden - "An attempt to find an expression for the distribution of fire damage amount"

As compared with the rectangular distribution assumed for the size of one claim by Johansen and Benktander in the previous papers, the joint authors of this paper have utilised a "condensed" Pareto function for this distribution.

The data, dwelling houses in the Southern part of Sweden, were subdivided into groups according to value, class of construction and quality of fire defence. The maximum destroyable value within a sub group is here denoted $s$. The amount of one claim was assumed to be distributed with the frequency function $p(z)$ where

$$
\begin{aligned}
p(z) & =(\alpha-\mathrm{x}) z^{-\alpha} & & \text { for } \mathrm{I} \leqslant z<\mathrm{s} \\
& =s^{1-\alpha} & & \text { for } a=\mathrm{s}
\end{aligned}
$$


(This function can be called a "condensed" Pareto function as the tail is "condensed" to the point corresponding to $z=s$ ).

$\alpha$ was estimated for each group by the method of maximum likelihood. (During the meeting Sternberg presented some corrected values of $\alpha$ which showed a monotonous increase with the code for fire defence (as would be expected) within each house-class).

Benckert, Sweden - "The premium in insurance against loss of profit due to fire as a function of the period of indemnity"

In this paper Benckert has fitted a "condensed" logarithmiconormal function to the frequency function of the period of interruption in insurance against loss of profit due to Fire. By assuming the loss frequency to be independent of the period of indemnity the author is able to confine his study to loss data only; further simplification is provided by the observations showing that the amount of loss per day of the period of interruption is almost constant.

The condensed logarithmico-normal function (with the point of condensation at the end of the period of indemnity) is fitted to the frequency function of the period of interruption, as found from the data, by the method of maximum likelihood, separately for industrial and non-industrial risks. Certain tests give support to the hypothesis that the function is essentially the same for the two risk groups.

It might be remarked that the formula used by the author for the mean amount of a claim can be transformed to a form commonly used in crop insurance. On very specialized assumptions regarding plant growth, the frequency function of the weight produced by the plant, which in the formula corresponds to the frequency function of the period of interruption, is given by a truncated logarithmico-normal function (cf. Philipson, Skand. Akt. Tidskr. I956, I-2).

Beard, England - "Analytical expressions of the risks involved in general insurance"

The results of this paper lend support to the conclusion that generally speaking condensed distributions should be used as expressions for the distribution of the amount of one claim in general insurance. 
The author has fitted two analytical expressions to data from Danish Fire Insurance for contents of buildings, covering the years I950-54. One of these expressions is a non-condensed logarithmiconormal curve and the other log-Pearson Type I, the latter being of finite range. The only criterion adopted was to secure the equality of the mean values of the actual and theoretical curves. Both expressions agree well with the data in the region investigated. The tails of the functions in the region, where data were absent, have been compared and are found to be strikingly different from a certain point. An excess of loss reinsurance is introduced and it is found that the two expressions differ very much in their effect on the moments of the claim distribution in the reinsurance. The differences are mainly due to the fact that the range of the logarithmico-normal curve was infinite, while that of the other curve was not. It is necessary to take account of the fact that the amount of one claim will never exceed a finite value.

Almer, Sweden - "Risk analysis in theory and practical statistics"

One part of the paper deals with a risk theory of quite a general scope. The basic conception of the theory is the risk situation - or potential accident - whether or not a claim or an accident actually occurs. Each risk situation constitutes a risk element associated with a pair of probabilities (specified for each situation), namely the probability that a claim shall occur, and the conditional probability on the hypothesis of a claim having occurred that the amount of the claim shall not exceed a specified value. The analysis is confined to finite sets of risk elements, characterised by certain measures of concentration. The maximum and minimum of symmetric functions of the probabilities concerned in a finite set of independent risk elements are given. The generalisation to non-independent risk elements is discussed.

In a second part of the paper it is proved that every distribution of the amount of one claim with finite range can be expressed by upper and lower exponential polynomials. Such approximations have also been obtained by graduating actual data. The transformation to the distribution of the aggregate amount of the claims, when the number of claims is at least approximately Poissondistributed, gives generalised Bessel-functions, which are reduced to 
standard expressions by the Borel-transformation. Three methods of calculation of the distribution of the aggregate amount of the claims are devised. In all simple problems the number of claims and amount of one claim may with a small alteration be exchanged in the expression for the distribution of the aggregate amount of claims. (A special case of this theorem is treated by Ammeter, Skand. Akt. Tidskr., I948). The ordinary risk theory based on the concept of a stochastic process with a transformed time parameter appears as a special case of the more general theory of Almer. It is further stressed in the paper that a statistical experiment for an insurance with risk fluctuations, with time and risk group, cannot be repeated. The theory afforded seems to entangle the difficulties implied in such risks.

The third part of the paper gives a summary of extensive statistical studies in Motor Car Insurance, involving compulsory third party and voluntary fire, theft and car damage insurance. The material is treated with the excess claim method, involving a partition of all claims into three classes according to the magnitude of the amount of the claims. Within each such class the $i^{\text {th }}$ moment about zero in the distribution function of the amount of one claim is approximately proportionate to the $i^{\text {th }}$ power of the mean of the distribution. It has further been found that the covariance between the number of claims and the amount of one claim is negligible. From this a simple expression is deduced for the mean error of the risk premium within each class. If the proportion between the number of claims in the two higher classes to that of all classes is used instead of the claim frequency in the two higher classes, or the proportion between the number in the highest class to that of the two higher classes is used instead of the frequency in the highest class, more stable measures are obtained, which may be used as a starting-point for equalisation. Within each class the variation of the number of claims and mean value of a claim, according to district, kind of vehicle, age of insurance and calendar year, can be approximately expressed by product factors, one for each basis of partition. The estimation of the factors has been based on the principle of Jeast squares and tested with the $\chi^{2}$-method. The sums of squares have been found to have a distribution function with certain similarities with the distribution of $\chi^{2}$ based on a normal 
deviate. Theoretically the distribution studied is represented by a series of terms of the same form as the distribution of $\chi^{2}$ for a normal deviate.

\section{Mattsson and Lundberg, Sweden - "Risk functions of Swedish sickness insurance"}

Formulae for capital values and premiums are given in terms of the sickness annuity technique, based on the concept of a probability of falling sick applied to the number of persons living instead of the number of actives. Analytical expressions for the intensity of falling sick and for the decrement function used in the bases of the sickness insurance are defined. Having regard to the developments by Almer it is interesting to note that these expressions have the form of exponential polynomials.

Ammeter, Switzerland - "Anwendungen der kollektiven Risikotheorie auf Probleme der Risikopolitik in der Sachversicherung",

In earlier works the author has developed methods of generalising the collective risk theory for application to general insurance. This generalisation is based on the negative binomial distribution. The development of the risk business is thus interpreted in terms of the Polya process, also studied by Ove Lundberg in his dissertation (Uppsala, I940).

The paper utilises the Polya process for dealing with a portfolio composed of different risks, mutually independent or dependent. When the loadings are sufficient for each risk group to ensure a certain level of the intensity $R$, implied in the main term of the probability of ruin, the author talks of "natural" calculation. As this method cannot, however, be used in practice, the author suggests that loadings shall be determined in order to ensure sufficient premiums for the total portfolio. The method is illustrated by numerical examples.

Philipson, Sweden - "On some distribution functions related to a specified class of stochastic processes"

Starting with a definition of the multivariate process, i.e. a process where a vector of parameters is substituted for the parameter of a univariate process, the author states that under certain 
conditions a multivariate process can be transformed to a univariate process. (Taking the opportunity to correct an error it might be remarked that in the second sentence of para. I on p. 265 of the paper the words "for every fixed value of $\omega_{\mathbf{1}}$ " shall be inserted between the words "which" and "either"). A class of processes is then defined, which is called the $H$ class, where $H$ is defined by $\mu_{1}(\mathbf{I}+H)=\mu_{2} ; \mu_{1}, \mu_{2}$ being the first two semi-invariants of the distribution of the number of changes in the random function attached to the process. A general expression for the probability of exactly $u$ changes, while the parameter $u$ passes from $u$ to $u+d u$, is given. It is shown that the Poisson, the Polya, the Arfwedson (Skand. Akt. Tisdkr. I955, I-2) process and the two processes which may be derived from the formulae given by Ammeter (Ibid., I948, 3-4) belong to the class. The distribution of the size of one change is, for the Poisson and the Polya process, assumed to be dependent on the parameter. These distributions, for all cases, are assumed to contain one absolutely continuous component, and to have finite moments to the $6^{\text {th }}$ order inclusive. On the condition that $H>-\frac{1}{2}$ and bounded, the distribution function of the normalised random function is expanded by the aid of a method given by Ammeter (1.c.) in an asymptotic expansion of which the usual expansion for the distribution of the gain (cf. Cramér, Skandia Jubilee Volume, Stockholm, I955) is a special case. Expansions for the distribution of the sum of squared deviates and the ratio between the two independent variance estimates are also given. (The index of $k$ under the summation sign in equation (I7) p. 274 of the paper should be $n+2 i)$.

Sparre Andersen, Denmark - "On the collective theory of risk in the case of contagion between the claims"

This paper is an application of risk theory to a special case of contagion between the claims.

The theory is based on the assumption that the probability of a claim in the time interval $t, t+\Delta t$ ( $t$ being the operational time) can be expressed as $\Delta t g(\tau)+o(\Delta t)$, when the preceding claim occurred at time $t-\tau$. Further a claim is simulated at time 0 to give a simple expression for the probability of the next claim. The choice of $g(\tau)$ is restricted, it should be non-negative, its 
integral between $o$ and I convergent and its integral between 0 and $\infty$ infinite. If $g(\tau)=$ constant the usual form of the collective risk theory is obtained. It is proved the probability of ruin fulfils an inequality of the usual form. The integral equation satisfied by this probability is also given. As an example definite forms of $g(\tau)$ and of the distribution of the amount of one claim are assumed which lead to simple formulae for the ruin probability. A numerical example shows that this probability is much higher when $g(\tau)$ is varying than in the case of constant $g(\tau)$ with the same loading.

Campagne with the collaboration of Driebergen, Netherlands"The influence of chain reactions on the loss distribution function"

A chain reaction is defined as "a process in which one event insured against, happening to a certain object, produces a number of similar events, which events in their turn cause similar events to once again other objects, etc.". It is said that in models previously used with dependent probabilities, as for instance the PolyaEggenberger process, the possible occurrence of chain reactions has not been taken into account.

For the further development this general concept is restricted to cases with the same degree of mutual dependency and with an alternative risk, i.e. that each event causes a total loss of the object in question. Utilising well-known properties of generating functions the authors deduce in an ingenious way necessary formulae for the different probabilities involved. The theory is applied to three models of the basic function of the chain reaction. The numerical calculations, carried out with the electronic computer ARMac, show the necessity of having regard to the influence of chain reactions, when judging the probability of ruin with available funds.

It might be remarked that, in case of fire insurance, the measurement of the contagion involved in the chain reactions is dependent on the definition of a risk. Suppose that we have to deal with a number of buildings, each building being a separate risk, a mutual contagion between the ignition frequencies of the buildings will then be revealed as such in the statistics of the number of claims. If, on the other hand, the risk is defined in such a way that several buildings will belong to each risk, the contagion between the fire 
frequencies with regard to each building within one risk will influence the frequencies of the amounts of one claim. Allowing for partial fires in each building this remark can be applicable to different parts of the same building.

A further remark will also be made. If the degree of mutual dependency is unequal for different risks involved the model will, as mentioned by the authors, be more complicated. In such a complicated model the process is probably not of the Markoff class, i.e. the probability of a claim occurring in a given short period of time depends not only on the status at the beginning of the period but also on the order of events before that time point. For such complicated models the Polya process is not applicable. When the model is simplified as much as the authors have assumed for deducing the probabilities involved, it might on the other hand be possible to apply Markoff models of more or less complicated nature. It could perhaps also be possible to use a model related to that described by Sparre Andersen in the paper reviewed above. In this connection it seems interesting to investigate whether Almer's remarks on dependent risk elements (Subject IVa, pp. 322-324) could be used to elucidate the interesting problem of chain reactions.

Thépaut, France - "Calcul du plein de conservation dans l'assurance des corps de navire"

Starting with an approximation to the probability of ruin based on the collective theory of risk, the author calculates the full amount of retention for French Marine Insurance, based on experience covering the years $195^{0-54}$. He finds the self-retention relative to the risk reserve for the different marine branches investigated to lie between $\mathrm{I} .36$ and 4.70 per cent with a probability of ruin of $10^{-6}$ and a profit coefficient of $10 \%$ of the risk premiums. For invalidity, non-industrial fire and industrial fire insurance the same computation gives $3.8,2.5$ and $2.1 \%$ respectively for the self-retention which, thus, is of the same order of magnitude for quite different branches of insurance. 